\title{
Estudio experimental in situ del comportamiento hídrico de un tramo del lienzo oeste de la Muralla de Ávila
}

\author{
María-Teresa Gil-Muñoz, Pedro-Pablo Pérez-García
}

\begin{abstract}
Resumen: El uso de sondas de tipo capacitivo es novedoso en el ámbito del patrimonio construido, por lo que se ha desarrollado una metodología exprofeso, con el fin de evaluar el comportamiento de la humedad en un muro de piedra, relleno de argamasa y piedras, correspondiente al tramo 44-45 del lienzo oeste de la muralla de Ávila. Con este estudio se demuestra la validez de las sondas FDR para analizar la influencia de los distintos aportes de agua en el muro, de precipitaciones y del subsuelo; así como la eficacia de las intervenciones realizadas en el mismo para paliar la humedad de capilaridad, como son la impermeabilización del adarve y la incorporación de drenajes a intramuros y a extramuros.
\end{abstract}

Palabras clave: fábrica de piedra, humedad de capilaridad, monitorización, sonda capacitiva FDR, análisis cualitativo

\section{Estudo experimental in situ do comportamento hídrico de um tramo ocidental da Muralha de Ávila}

Resumo: O uso de sondas do tipo capacitivo é uma novidade no campo do património edificado, para o qual foi desenvolvida uma metodologia expressa, a fim de avaliar o comportamento da humidade numa parede de pedra, argamassa e pedras, correspondente ao item 44-45 do tramo ocidental da parede medieval de Ávila. Este estudo demonstra a validade das sondas FDR para analisar a influência das diferentes contribuições da água na parede, da precipitação e do subsolo; bem como a eficácia das intervenções nele realizadas para o alívio da humidade por capilaridade, como a impermeabilização e a incorporação de drenos intramuros e extramuros.

Palavras-chave: fábrica de pedra, humidade capilar, monitoramento, sonda capacitiva FDR, análise qualitativa

\section{An in situ experimental study of the hydric behaviour of a section at the western stretch in the medieval wall in Ávila}

\begin{abstract}
The use of capacitive type probes is new in the field of built heritage, so a specific methodology has been developed, in order to evaluate the behaviour of moisture in a stone masonry wall, filled with mortar and stones, corresponding to section 44-45 at the western stretch in the medieval wall in Ávila. This study shows the value of the FDR probes to analyse the influence of the water input on the wall, from the rainfall and subsoil; as well as the efficiency of the interventions carried out on the same wall to cut the rising damp, such as the waterproofing of the adarve (walkway on the ramparts) and the incorporation of drainage facilities inside and out next to the wall.
\end{abstract}

Keyword: stone masonry, moisture, monitoring, FDR capacitive probe, qualitative analysis 


\section{Introducción}

El presente estudio queda enmarcado en el proyecto de "Consolidación y restauración de la muralla de Ávila, correspondiente a los lienzos 84-85 al 87-1, incluidos los cubos $85,86,87,81$ y 4 y actuaciones para prevenir las humedades en parte del lienzo este de la muralla de Ávila", redactado en agosto de 2016.

La obra fue promovida por el Ayuntamiento de Ávila, financiada por el Ministerio de Fomento (actual Ministerio de Transportes, Movilidad y Agenda Urbana), y ejecutada por la empresa Velarenas entre 2016 y 2017. La empresa Velarenas (Barba Grupo Inmobiliario) encargó esta investigación para evaluar in situ el comportamiento hídrico de la muralla, entre junio de 2017 y mayo de 2019, tras la intervención del tramo 44-45 en el lienzo oeste (GilMuñoz 2017). Este tramo presentaba grandes manchas de humedad y sales, tanto a intramuros como a extramuros.

Ávila es una ciudad del interior de la Península Ibérica cuya muralla es Monumento Nacional desde 1884 y Patrimonio de la Humanidad desde 1985; el tramo 44-45, objeto de este estudio experimental, se localiza en la parte baja de la ciudad medieval, próxima al río Adaja [figura 1].

La ciudad se sitúa al norte del Sistema Central en el Valle de Amblés en un altozano granítico a $1130 \mathrm{~m}$ de altitud, con un clima oceánico mediterráneo, la amplitud térmica diaria es alta (promedio anual de $27^{\circ} \mathrm{C}$ ) y el régimen de precipitaciones es bajo (400 mm/año).

En este lugar ha habido múltiples asentamientos desde el Paleolítico a la Edad del Hierro. También hay evidencias arqueológicas de poblamientos de cierta entidad, vetones, romanos (sin categoría de municipio) y visigodos. Aunque siempre se ha especulado sobre el posible origen romano de la muralla, hasta las últimas excavaciones realizadas, con motivo de diversas intervenciones de restauración en la misma, no se ha podido confirmar de manera definitiva la existencia de una muralla de época romana. De hecho, la excavación arqueológica llevada a cabo en este tramo ha servido para corroborar tal extremo (Ruiz y Cabrera 2017).
La muralla de Ávila tiene $2,5 \mathrm{~km}$ de longitud, con una altura variable según las posibilidades defensivas de cada tramo, y $3 \mathrm{~m}$ de ancho. Se encuentra íntegra, vertebrando el trazado medieval de la ciudad tanto a intramuros como a extramuros. Es seña de identidad de la ciudad desde antiguo, como así lo demuestran la literatura, los cuadernos de viajeros, los grabados, las fotografías, las pinturas e incluso el cine.

Gran parte del adarve es visitable y en torno a la muralla se celebran múltiples actividades culturales, dirigidas tanto a los habitantes de la ciudad como a sus visitantes, en este sentido destaca por su popularidad el mercado medieval que se celebra el primer fin de semana de septiembre. Este monumento es el más visitado de la ciudad, y los últimos datos de febrero de 2020 indican un aumento de las visitas a la muralla, con el consiguiente incremento de la facturación (La Vanguardia 2020).

La humedad de capilaridad es uno de los factores que afectan a la conservación de la muralla de Ávila, cuestión que justifica esta investigación.

La técnica utilizada para medir la humedad en el muro ha sido la reflectometría de dominio de frecuencias (FDR, acrónimo del término inglés). Consiste en medir el comportamiento dieléctrico de los materiales del muro a través de sensores de capacitancia. Estas sondas son de uso habitual en el campo de la agricultura (por ejemplo, para control del riego), pero su aplicación es novedosa en el ámbito del patrimonio construido.

Se han instalado 8 sondas de humedad en el muro, conectadas a dos registradores con transmisión remota de datos, que ha permitido el seguimiento en tiempo real del régimen hídrico de este tramo de la muralla.

La metodología desarrollada exprofeso ha consistido en el ensayo y la calibración de las sondas en laboratorio, la instalación de las sondas en la sección del muro en superficiey a distinta profundidad (extracción de mortero, inserción de las sondas, retacado del mortero y sellado), así como la sujeción y disimulo estético del cableado y de los registradores.

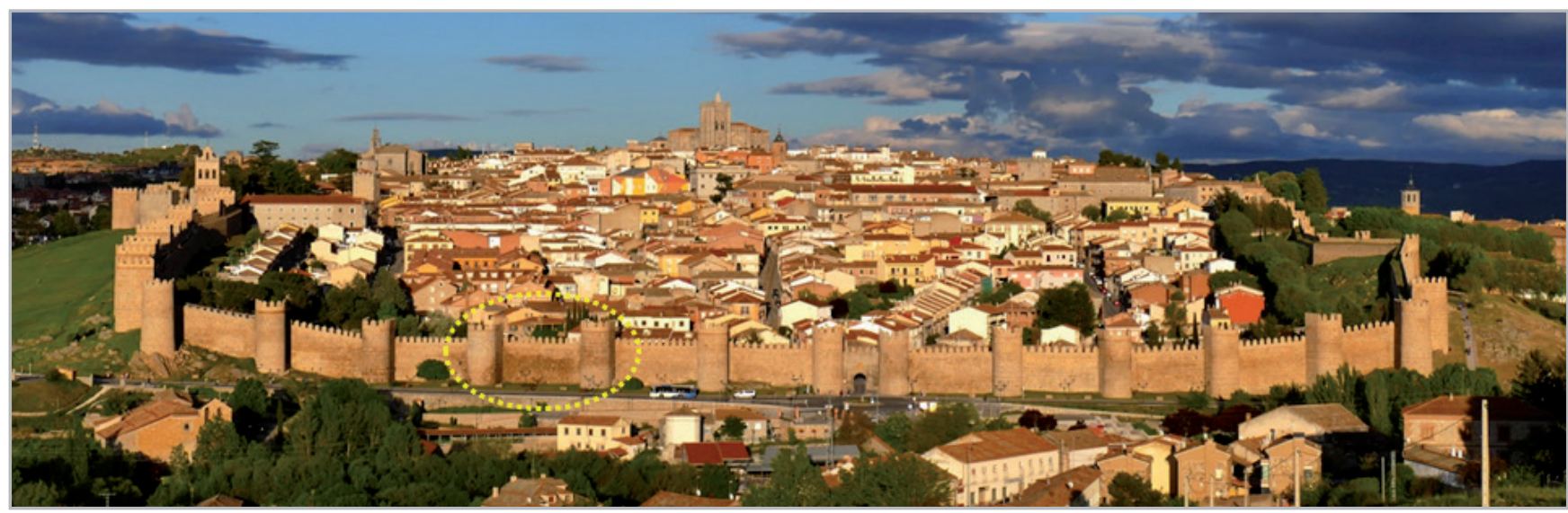

Figura 1.- Vista oeste de la ciudad medieval de Ávila, 2020-05-11. El círculo indica la situación del tramo 44-45 de la muralla. 
En laboratorio se han ensayado distintas muestras de morterosy suelos, extraídas durante las obras de excavación y arqueología necesarias para la implementación de las intervenciones proyectadas de impermeabilización y de drenaje. Se ensayó la humedad de los morteros en donde iban a quedar instaladas las sondas, así como la presencia de sales (tipo y concentración) en los mismos. También se estudió la influencia de distintos factores en la medición de las sondas FDR: compactación del material en contacto con las sondas, presencia de sales en los morteros y temperatura ambiente.

En campo se ha constatado el interés de las sondas FDR para medir el comportamiento hídrico del muro, sin obviar los factores que afectan a la medición, como son la presencia de sales y la temperatura ambiente. Las sales no han sido determinantes en las mediciones puesto que se está lejos de los umbrales establecidos por el fabricante. Y la temperatura ambiente es algo que se puede sortear, a través del análisis de los datos.

\section{Estado del arte}

Distintos autores han hecho una revisión teórica sobre las técnicas de medición del contenido de humedad en los materiales y en los muros de las fábricas antiguas (Krus 1996: 33-38; Tada y Watanabe 1998; McCann y Forde 2001; Healy 2003; Voutilainen 2005; Saïd 2007; Phillipson et al. 2007; Pinchin 2008; Tiano y Riminesi 2017; Camuffo 2018; Agliata et al. 2018). Además, hay una norma europea en este sentido, la UNE-EN 16682:2018. En la literatura también queda recogida la práctica de estos métodos en laboratorio (Sandrolini y Franzoni 2006; Torres y Freitas 2010; Camino et al. 2014). Del mismo modo hay artículos sobre la experiencia que se tiene en campo (García-Morales et al. 2012; MartínezGarrido et al. 2015; Hoła et al. 2017; Vecchiattini 2018).

Entre las diferentes técnicas para medición de la humedad en los materiales se encuentran las electromagnéticas (Regalado et al. 2009; Kaatze y Hübner 2010): de reflectometría de dominio temporal (TDR, acrónimo del término inglés Time Domain Reflectometry), de tipo capacitivo o reflectometría de dominio de la frecuencia (FDR, acrónimo del término inglés Frecuency Domain Reflectometry) e impedancia. Se diferencian en el principio físico de funcionamiento: tiempo de tránsito, capacitancia e impedancia. Tanto las sondas TDR como las FDR son técnicas dieléctricas (Zhao et al. 2012) que permiten medir el contenido de humedad volumétrico.

En cuanto a las sondas TDR hay muchas referencias para el estudio y caracterización de los suelos en el ámbito de la geología y de la agricultura. También hay estudios teóricos (Hauschild y Menke 1998; Pavlík y Černý 2004; Phillipson et al. 2008; Černý 2009; Mollo y Greco 2011; Suchorab et al. 2016; Cataldo et al. 2018) y experimentales en laboratorio (Agliata et al. 2016; Freitas et al. 2020) en el campo del patrimonio. Esta técnica admite mediciones puntuales y en continuo.
Respecto de las sondas de tipo capacitivo apenas hay bibliografía a nivel teórico o experimental en el campo del patrimonio construido. Aznar-Mollá (2016) propone mediciones puntuales repetidas en el tiempo en la superficie del paramento objeto de análisis (definida una malla virtual) para diagnóstico de la humedad de capilaridad. En la presente investigación se plantea el uso de las sondas FDR, más económicas y de uso menos complejo que las TDR, con el fin de disponer de mediciones en continuo, para estudio de la evolución de la humedad en el muro en superficie o en profundidad.

\section{Objetivo}

Con este estudio se pretende conocer la evolución de la humedad en el muro (adarve, relleno de la muralla, paramentos verticales y base del muro) después de implementar determinadas medidas para mitigar la incidencia del agua (filtraciones y capilaridad) en función de su origen (pluviales, escorrentías y riego); en definitiva, se trata de testar la validez y eficacia de los sistemas constructivos adoptados (impermeabilización y drenajes).

Los objetivos específicos de la investigación se concretan en los siguientes puntos:

- Estudiar la evolución del contenido de humedad volumétrico (VWC, acrónimo del término inglés Volumetric Water Content), expresado en \%.

- Observar si se producen filtraciones a través del adarve o de la mampostería exterior (por precipitaciones y viento).

- Estimar si tienen lugar fenómenos de capilaridad desde el suelo (pluviales, escorrentía y riego).

\section{Metodología}

El tramo 44-45 de la muralla, situado en la parte baja de la ciudad, está solado intramuros y ajardinado con riego extramuros. Intramuros se encuentra un pozo con agua [figura 2a] y en el lado norte contiguo hay un jardín, con vertido de agua a extramuros [figura 2b]. La muralla está asentada sobre roca de granito. La cota del suelo intramuros es superior a la cota del suelo extramuros.

El muro del tramo objeto de estudio está formado por dos hojas de granito enripiado (constituidas por hiladas de piedras puestas a espejo, sobre todo en la parte inferior) y un relleno de argamasa y piedras. El adarve está solado con pendiente hacia una canaleta en la cara interior del muro.

En su cara interior y sobre todo en su cara exterior, el muro muestra manchas de humedad y de sales (Fort et al. 2015), cuya intensidad y extensión fluctúan a lo largo del año (mayor en invierno que en verano). 


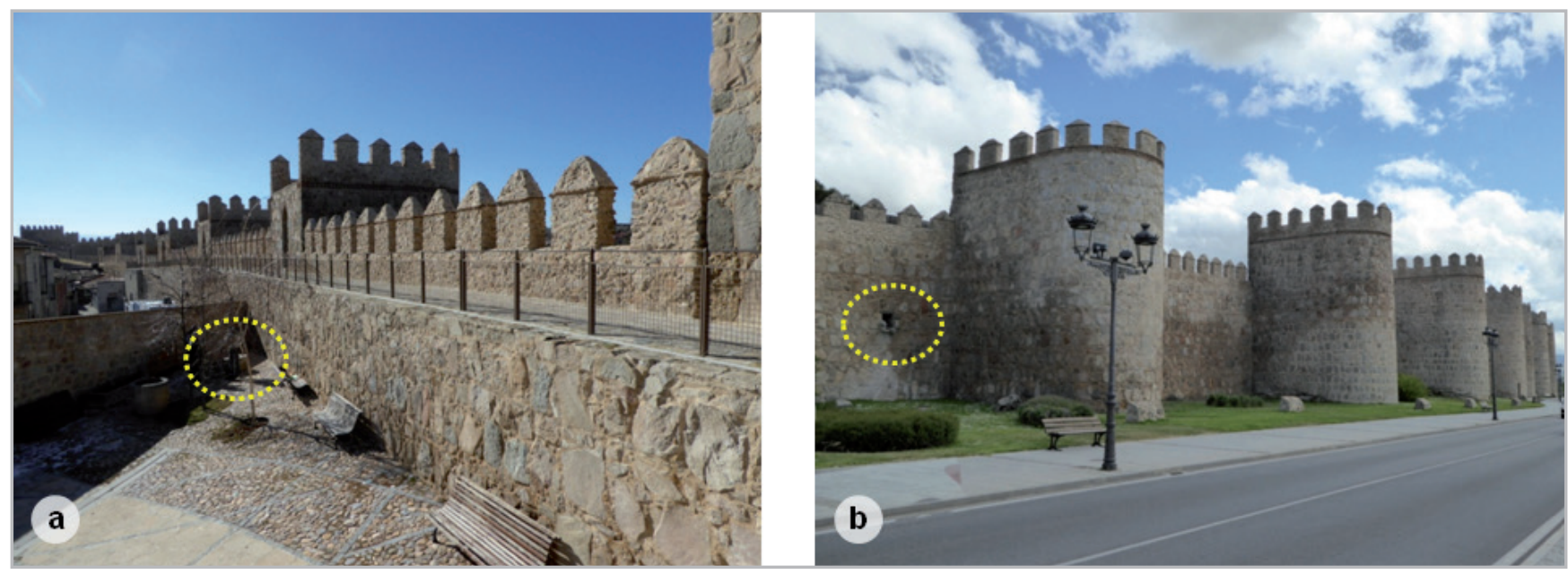

Figura 2.- Tramo 44-45: a) Intramuros, 2017-01-24. El círculo indica la situación de un pozo; b) Extramuros, 2017-05-10. El círculo indica la evacuación de una atarjea en el tramo contiguo.

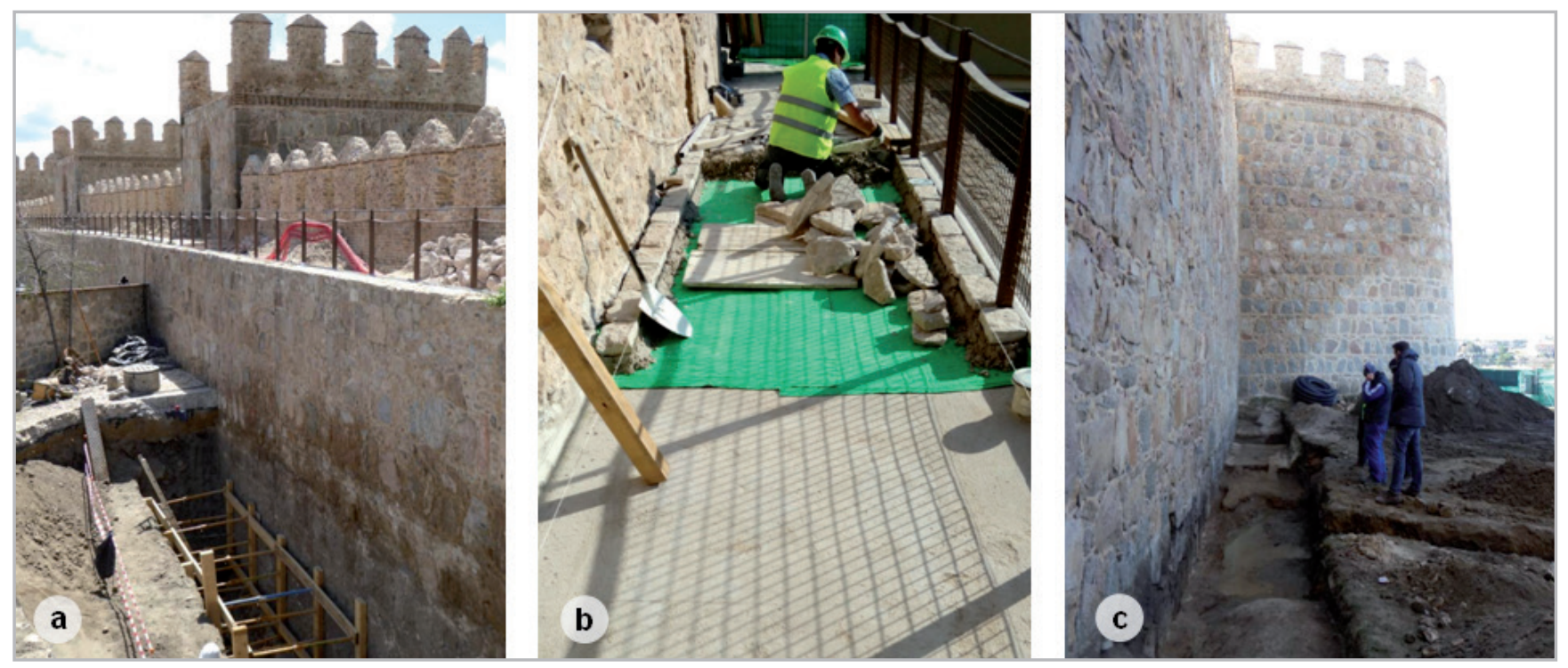

Figura 3.- Actuaciones en el tramo 44-45: a) Intramuros, 2017-05-10; b) Adarve, 2017-06-01; c) Extramuros, 2017-01-24.

Las intervenciones llevadas a cabo en el marco de esta investigación, según los distintos aportes de agua que recibe el muro (precipitaciones, escorrentía y riego), consistieron en la impermeabilización del solado del adarve y en la colocación de drenajes del suelo a intramuros (a la altura de la sonda HS1) y a extramuros (por encima de la sonda HS3) [figura 3 y figura 4].

\section{-Técnica de medición FDR}

De las distintas técnicas conocidas para medición de la humedad en los materiales, las que parecían más adecuadas para el fin del presente estudio eran las técnicas electromagnéticas.

Se optó por la sonda de tipo capacitivo FDR, por su menor precio y la posibilidad de registro continuo, con el reto de que no se conocían estudios sobre la aplicación de esta técnica en el patrimonio cultural inmueble, al contrario que con las sondas de reflectometría de dominio temporal (TDR) cuya aplicación en laboratorio es muy reciente.

El principio de funcionamiento de las sondas FDR es medir la permitividad dieléctrica de los materiales (es decir, su habilidad para soportar una carga eléctrica). La permitividad dieléctrica está en relación directa con el contenido de humedad volumétrico (VMC, acrónimo del término inglés Volumetric Water Content), expresado en $\%$. Se trata de una medida indirecta del contenido de humedad volumétrico (que se define como la relación existente entre el volumen de agua y el volumen de muestra), realizada in situ, frente a la medida directa en laboratorio (mediante pesadas en fresco y en seco de una muestra de volumen conocido) que requiere de una toma de muestra. 
Por tanto, se pretendía medir el comportamiento dieléctrico de los materiales del muro a través de sensores de capacitancia FDR, que se basan en la técnica de reflectometría de dominio de frecuencias.

Las características de la sonda de tipo capacitivo utilizada, en concreto del modelo EC-5 (formado por un mango con dos palas, marca Decagon Devices), son las siguientes: las medidas son $10 \mathrm{~cm}$ de largo y $2 \mathrm{~cm}$ de ancho (requieren una perforación de $3 \mathrm{~cm}$ de diámetro para su instalación). Su rango de medición es del 0-100 $\%$ de contenido de humedad volumétrico (como están calibradas para suelos, puesto que su uso habitual es el de control del riego en la agricultura, la cantidad máxima de agua que edafológicamente puede tener un suelo es del $40 \%$ aproximadamente). La precisión del sensor es del 3 $\%$. Y su volumen de exploración es de 0,3 I (equivalente a un cilindro de $9 \mathrm{~cm}$ de diámetro aproximadamente). La durabilidad de las sondas está supeditada a la oxidación de las palas o a factores externos como la rotura de cables (por ejemplo, por roedores).

Se ha optado por una medición cualitativa, puesto que se trata de observar la evolución de la humedad en el muro en el tiempo. En cualquier caso, la medición se ve afectada por la temperatura del material en contacto con la sonda y la conductividad eléctrica del material debida a la presencia de sales. Por tanto, previo a la instalación de las sondas se realizó un ensayo de los morteros y suelos en laboratorio; también se testaron los equipos en campo mediante medidas directas (o lecturas en tiempo real).

\section{- Ensayo en laboratorio}

Una vez se seleccionó el modelo de la sonda (EC-5) que se podía adaptar a nuestro objetivo, se ensayaron las muestras de morteros y suelos en laboratorio y se calibraron las sondas. Para ello se utilizó un equipo de lectura en tiempo real.

Se ensayaron distintas muestras de los morteros y suelos que iban a quedar en contacto directo con las sondas. Partiendo de una fase en seco se hizo una clasificación del rango de valores en los que el material se podía considerar en estado seco (0-20\%), húmedo (20-3 0\%) y mojado (30$40 \%$ ). El valor máximo es el del agua, siendo su valor del $60 \%$. También se evaluaron otros factores que influyen en las mediciones: los cambios de la temperatura ambiente y la presencia de sales en los materiales. Se constató que una medición cualitativa permitiría alcanzar el objetivo de la investigación, es decir, conocer la fluctuación de la humedad en el muro según las distintas acciones del agua. Una vez testadas las sondas en laboratorio, se colocaron in situ, haciendo comprobaciones de las mediciones en tiempo real, antes de que estas quedaran conectadas a los registradores o loggers.
Los registradores son equipos de medición autónoma que almacenan la información medida a intervalos programados y que podemos visualizar en remoto, ya que en este caso se dispone de un módem GPRS que envía los datos a un servidor central al que accede el usuario.

\section{— Instalación de los equipos}

La instalación de este aparataje se realizó en el momento de ejecución de las obras proyectadas. En total se instalaron 8 sondas de humedad y 2 registradores, uno intramuros (LI) y otro extramuros (LE), como se puede apreciar en la sección de la imagen [figura 4]. Al primer registrador se conectaron 5 sondas (3 intramuros y 2 en el adarve), y al segundo registrador extramuros se conectaron 3 sondas. Las sondas se colocaron en superficie y a distinta profundidad en el adarve, intramuros y extramuros.



Figura 4.- Localización de las sondas de humedad del suelo HS y los registradores o loggers $L$ en la sección del muro. En color se representa la mancha de humedad a extramuros e intramuros.

Todas las sondas se encuentran en la misma sección transversal del lienzo, en la mitad del tramo 44-45, salvo la sonda HS5 bajo el solado del adarve, desplazada 1,5 m hacia el lado del pozo.

El mortero de cada perforación (alterado mínimamente) se recogió para el posterior retacado de la sonda tras su colocación. Se aseguró un buen contacto de la sonda (en las palas y el mango de cada sonda) con el material a medir, evitando la presencia de piedras o bolsas de 

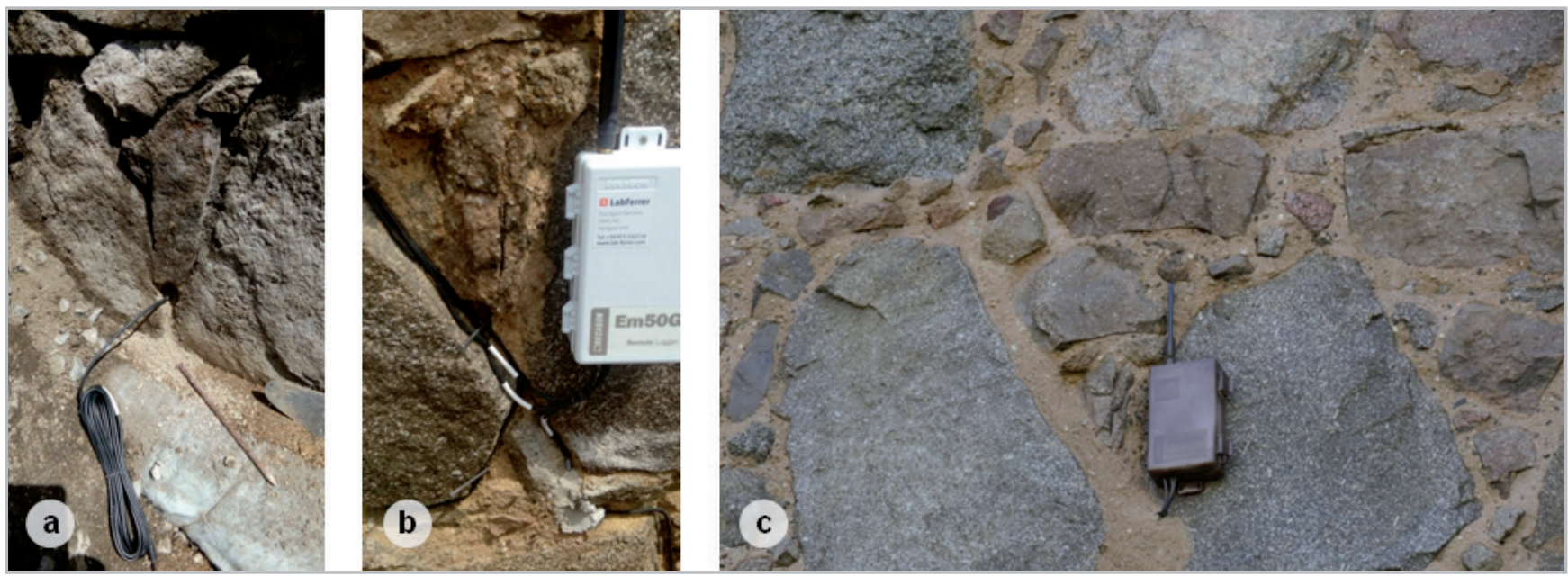

Figura 5.- Colocación de las sondas HS1, HS8 y el registrador LE, 2017-06-01.

aire, procurando el mismo grado de compactación en el material retacado. El material sobrante se guardó para analizar en laboratorio su contenido de humedad gravimétrico, conductividad y tipo de sales presentes en cada muestra.

Los cables se ocultaron con mortero sobre las rozas realizadas previamente en el llagueado de la fábrica, pintándose los registradores para disimular su presencia [figura 5].

Tras la colocación de las sondas se aguardó un periodo de estabilización, debido a la humedad del mortero de sellado, el acabado de las actuaciones proyectadas y a las nuevas condiciones del medio.

\section{- Análisis en campo}

A través de los datos medidos en campo, se comprobó la incidencia de los distintos factores relacionados con la humedad, como son: la estabilización de las mediciones tras el retacado y sellado de los morteros que aseguran las sondas (se aprecia en los sensores a intramuros y a extramuros); la ejecución de las intervenciones previstas que también suponen un aporte de humedad (como en la sonda HS5 tras la impermeabilización y solado del adarve); y las precipitaciones, con influencia en todas las sondas salvo en las que se encuentran a más profundidad (como la HS6 en el relleno o la HS7 extramuros) o protegidas mediante impermeabilización (como la HS1 extramuros) [figura 6]. En cualquier caso,

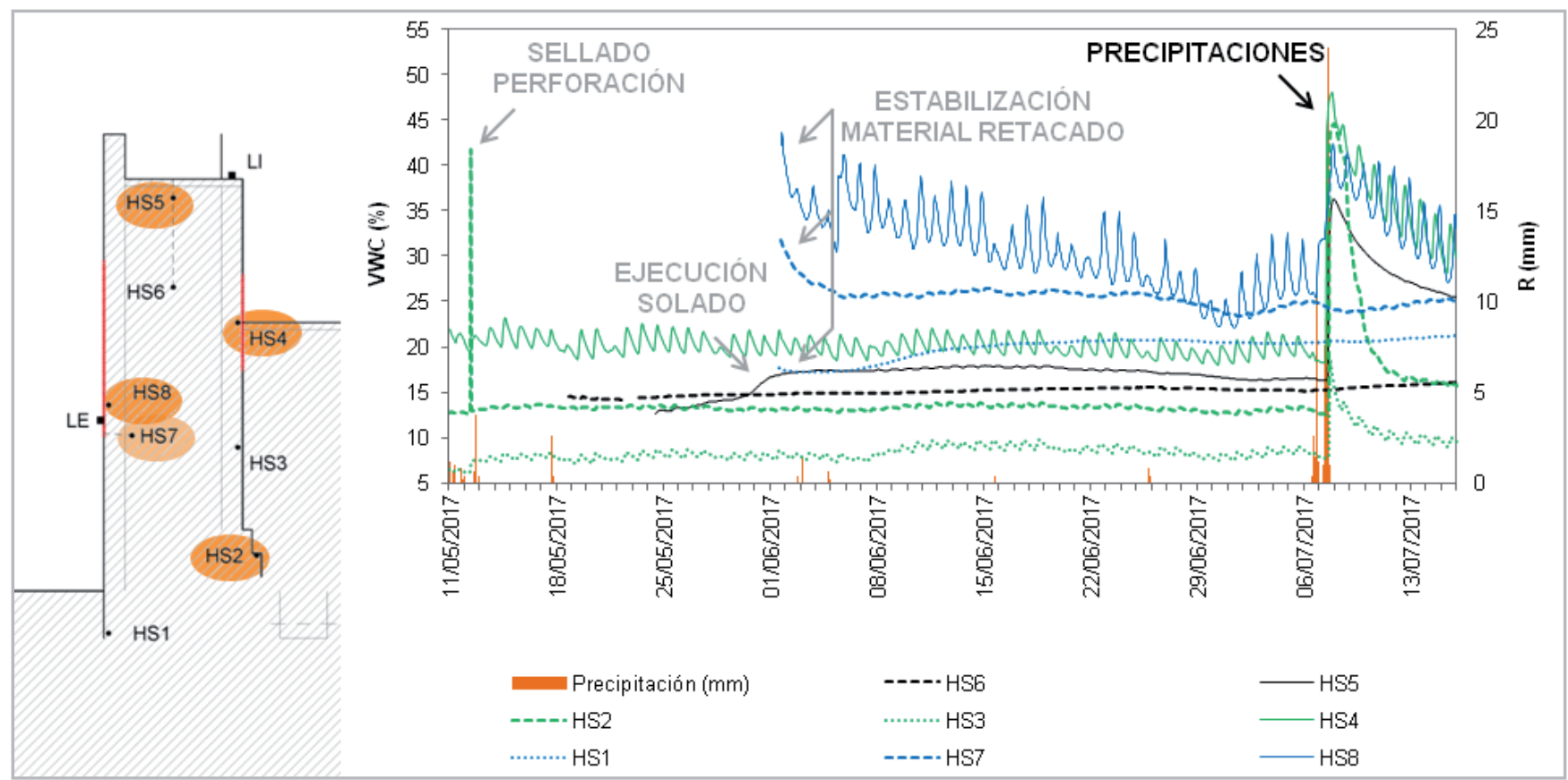

Figura 6.- Influencia de la humedad en las mediciones. En la gráfica las curvas en azul se corresponden con las mediciones extramuros, en verde con las mediciones intramuros y en negro con las mediciones del adarve; las precipitaciones han sido registradas por el observatorio de la Agencia Estatal de Meteorología (AEMet) en Ávila (<http://www.aemet.es $>)$. En la sección quedan identificadas las sondas afectadas, con diferente intensidad. 


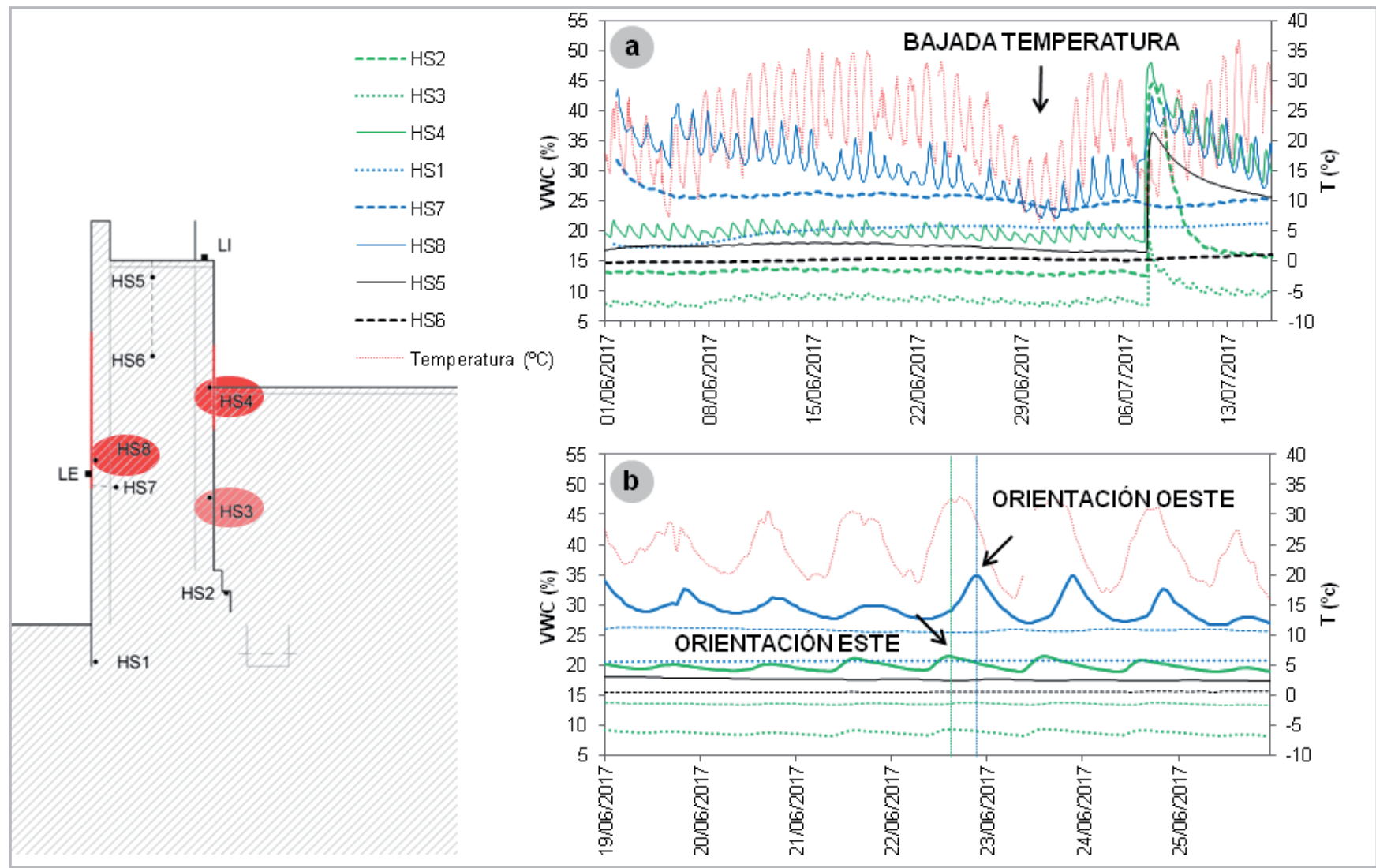

Figura 7.- Influencia de la temperatura en las mediciones. En las gráficas la curva en rojo se corresponde con la temperatura del clima local: a) Periodo de mes y medio; b) Periodo de una semana. En la sección quedan identificadas las sondas afectadas, con diferente intensidad.

tras un periodo de precipitaciones las sondas necesitan un tiempo de estabilización más o menos dilatado (según las condiciones de contorno o el grado de permeabilidad de los materiales).

También influyen en las mediciones los cambios de temperatura del material que queda en contacto con la sonda. Según el fabricante, la variación del contenido de humedad volumétrico es del $0,5 \%$ por cada $1{ }^{\circ} \mathrm{C}$.

En este caso los dos paramentos de la muralla (del tramo 44-45 del lienzo oeste) están expuestos a insolación. A mayor exposición de las sondas mayor afección en las mediciones (como ocurre en las sondas HS8 extramuros y HS4 intramuros), en cuyas mediciones se registran los ciclos diarios de temperatura, también se puede apreciar el cambio de temperatura en el transcurso de los días [figura 7, gráfica superior]. Del mismo modo, las sondas con orientación oeste están más afectadas que las sondas con orientación este (la excavación intramuros estuvo abierta hasta abril de 2019) [figura 7, gráfica inferior].

\section{Resultados y discusión}

Se ha comprobado, en laboratorio y en campo, que las sondas FDR son válidas en la monitorización de muros, quedando en contacto con los morteros de rejuntado o argamasas de relleno. Del análisis de los datos se desprende la influencia de las sales de los morteros y la evolución de la humedad en el muro.

\section{- Presencia de sales en los morteros}

Las mediciones de las sondas electromagnéticas se ven afectadas por la conductividad eléctrica de los morteros si la presencia de sales es muy elevada. El fabricante indica que la sonda EC-5 es precisa hasta los $8000 \mu \mathrm{S} / \mathrm{cm}$, y este dato no se ha superado en ninguna de las muestras analizadas.

La mayor concentración de iones ha tenido lugar en la superficie del paramento con orientación oeste (con una conductividad eléctrica media de $560 \mu \mathrm{S} / \mathrm{cm}$ y cierto contenido en sales). Esta distribución de sales era mayor en superficie respecto a la profundidad $(50 \mathrm{~cm})$ del muro. La intensidad de la mancha y su extensión en este paramento es cambiante con las estaciones meteorológicas.

En el paramento con orientación este la conductividad eléctrica era más baja; a nivel de suelo la conductividad era muy baja; y en el adarve y relleno era prácticamente nula. En cualquier caso, el nivel de concentración de sales en los puntos de medición no ha afectado a la lectura de las sondas EC-5. 


\section{— Evolución de la humedad en el muro}

La medición cualitativa ha permitido observar el comportamiento hídrico de la muralla, vinculándose estos cambios fundamentalmente a las precipitaciones y a los aportes de humedad del subsuelo.

De manera específica la evaluación de los datos ha servido para valorar la eficacia de los sistemas constructivos adoptados para paliar la humedad en el muro en este tramo de la muralla.

Mediante la monitorización, el análisis e interpretación de los datos se ha constatado que:

1. En el adarve (los sensores no están afectados por la insolación), la humedad queda retenida por la lámina de impermeabilización incorporada en la restauración del solado (se aprecia en la curva superior de la sonda HS5) y conducida a la canaleta interior, por tanto, la humedad no afecta a los rellenos del muro (como se demuestra con la curva inferior de la sonda HS6) [figura 8]. Esta gráfica y las dos siguientes se corresponden con un ciclo anual completo, en concreto al año 2018 cuyo primer semestre fue especialmente lluvioso (casi el doble de lo normal); y se representan valores promedios diarios, para atenuar el efecto de la temperatura en las sondas que se ven afectadas por la insolación.

2. Extramuros hay 3 sondas [figura 9]. La inferior (HS1), tras la impermeabilización del drenaje del jardín con riego, desde su instalación en mayo de 2017 se muestra en estado seco incluso durante el primer semestre lluvioso de 2018, y trascurrido el verano empezó a aumentar de manera



Figura 8.- Gráfica de las mediciones registradas por las sondas de humedad colocadas en el adarve (según se indica en la sección de la izquierda) y de las precipitaciones registradas por el observatorio de la AEMet en Ávila (<http://www.aemet.es $>)$.



Figura 9.- Gráfica de las mediciones registradas por las sondas de humedad colocadas extramuros (según se indica en la sección de la izquierda) y de las precipitaciones registradas por el observatorio de la AEMet en Ávila (<http://www.aemet.es $>$ ). 


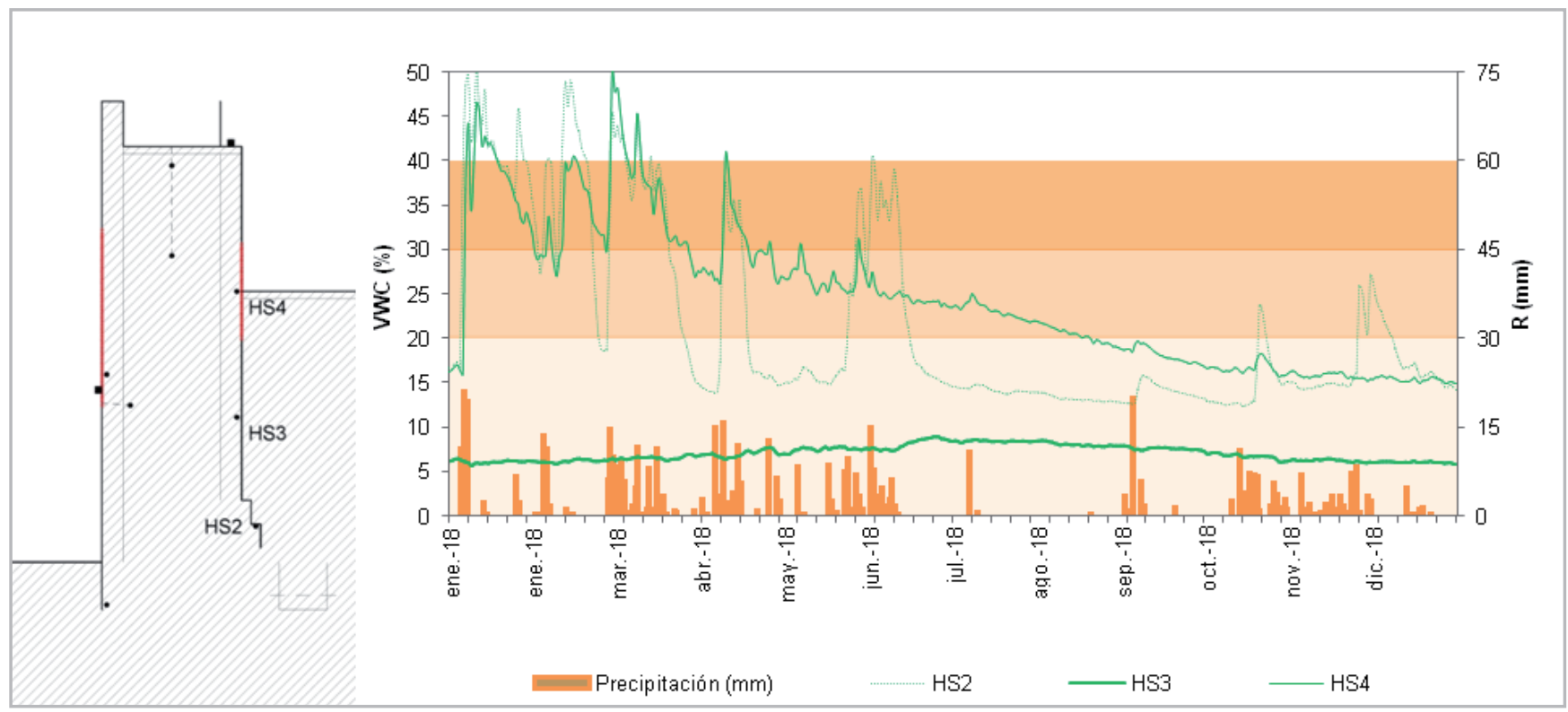

Figura 10.- Gráfica de las mediciones registradas por las sondas colocadas intramuros (según se indica en la sección de la izquierda) y de las precipitaciones registradas por el observatorio de la AEMet en Ávila (<http://www.aemet.es $>$ ).

progresiva el contenido de humedad volumétrico hasta quedar en estado mojado.

La sonda situada en la superficie extramuros (HS8) muestra la influencia de las precipitaciones, por acción de los vientos dominantes, que en Ávila son de dirección noroeste-sureste. En ausencia de precipitaciones la superficie del muro se encuentra en estado seco.

El sensor colocado a $50 \mathrm{~cm}$ de la superficie del muro (HS7) se vio afectado por las lluvias intensas de marzo a junio de 2018, estabilizándose posteriormente en su estado inicial, siendo esta su fase normal.

3. Intramuros también hay 3 sondas [figura 10], desde el nivel de suelo hasta la cimentación (de origen romano según se constató tras la excavación). Las mediciones de la gráfica se corresponden al periodo en que la excavación intramuros permaneció abierta. El sensor inferior (HS2) recoge la acumulación del agua precipitada en forma de lluvia o nieve. La sonda en la superficie intramuros a nivel del suelo (HS4), al igual que la sonda en la superficie extramuros, se vio afectada por las precipitaciones intensas del primer semestre del año, siendo su estado normal seco. La sonda en la superficie intramuros por debajo del nivel del suelo (HS3), protegida de los vientos dominantes, siempre se ha mantenido estable, en estado seco. La excavación se cerró en mayo de 2019 incorporando un drenaje a la altura de la sonda HS3, que cambió su estado de seco a mojado con las lluvias del otoño del 2020.

Los paramentos verticales se ven afectados por las precipitaciones según los vientos dominantes, llegando al menos a una profundidad de $50 \mathrm{~cm}$ en periodos de precipitación intensa y racheada.

\section{Conclusiones}

Como conclusión se demuestra el interés de las sondas electromagnéticas de tipo capacitivo para seguimiento del comportamiento hídrico real de los muros sometidos a humedad capilar, y para evaluación de la eficacia de las medidas implementadas para su conservación.

En el análisis e interpretación de los datos se ha de evaluar y considerar la presencia de sales en los morteros o suelos objeto de estudio, así como la influencia de la temperatura ambiente en las mediciones.

En el caso del tramo 44-45 del lienzo oeste de la muralla de Ávila el nivel medio-bajo de concentración de sales existente en los distintos puntos de medición no ha alterado los valores registrados a través de las sondas FDR.

Sin embargo, todas las mediciones de las sondas ubicadas en la superficie del muro quedan alteradas por la fluctuación de la temperatura ambiente, en mayor medida cuando los sensores están expuestos a la radiación del sol, en este caso con orientaciones este y oeste. Esta cuestión se ha sorteado trabajando con valores promedio.

En cuanto a la evolución de la humedad en la sección del muro del tramo 44-45 se ha constatado la incidencia de las precipitaciones y de la humedad del subsuelo. En el adarve la humedad queda retenida por la lámina de impermeabilización incorporada; los paramentos verticales (no intervenidos) se ven afectados por las precipitaciones según los vientos dominantes; y en la base del muro, junto a los tubos drenantes, los valores de humedad permanecen estables hasta que transcurrido un año de su instalación tienen lugar las primeras precipitaciones del otoño alcanzando el estado de mojado. Este último aspecto 
requiere de un seguimiento que permita valorar de manera más clara la eficacia de los drenajes.

Respecto a las manchas de humedad se mantienen en ambas caras del muro, una vez impermeabilizado el adarve o drenado el suelo intramuros y extramuros. Se manifiestan con distinta intensidad y extensión a lo largo del año, probablemente vinculadas a la condensación higroscópica de las sales (mayor en el paramento extramuros).

\section{Agradecimientos}

A Cristina Sanchidrián Blázquez, Pilar Fernández Valdés y Rosa Ruiz Entrecanales (dirección facultativa por parte del Ayuntamiento de Ávila); a José Luis López-Hernández (Construcciones Velarenas, Barba Grupo Inmobiliario); a Javier Jimeno Jimeno y Óscar Romo Caballero (jefes de obra); a Óscar, Nacho y Diego (encargados de obra); y a Blas Cabrera González (Castellum S. Coop.).

\section{Referencias}

AENOR (2018). UNE-EN 16682 Conservación del patrimonio cultural. Métodos de medición del contenido de humedad, o contenido de agua, en materiales constitutivos del patrimonio cultural inmueble.

AGLIATA, R., GRECO, R. y MOLLO, L. (2018). "Moisture measurements in heritage masonries: A review of current methods", Materials Evaluation, 76(11): 1468-1477. https://www.researchgate. net/publication/325285165

AGLIATA, R., MOLLO, L. y GRECO, R. (2016). "Use of TDR to compare rising damp in three tuff walls made with different mortars", Journal of Materials in Civil Engineering, 29(4). https:// doi.org/10.1061/(ASCE)MT.1943-5533.0001794

AZNAR MOLLÁ, J.B., BENLLOCH MARCO, J. y MEDINA RAMÓN, F.J. (2016). El diagnóstico de las humedades de capilaridad en muros y suelos. Determinación de sus causas y origen mediante una metodología basada en la representación y análisis de curvas isohídricas. Tesis doctoral, Universidad Politécnica de Valencia. https://doi.org/10.4995/Thesis/10251/61630

CAMINO, M.S., LEÓN, F.J., LLORENTE, A. y OLIVAR, J.M. (2014). "Evaluation of the behavior of brick tile masonry and mortar due to capillary rise of moisture", Materiales de Construcción, 64(314): e020. https://doi.org/10.3989/mc.2014.02513

CAMUFFO, D. (2018). "Standardization activity in the evaluation of moisture content", Journal of Cultural Heritage, 31: S10-S14. https://doi.org/10.1016/j.culher.2018.03.021

CATAlDO, A., DE BENEDETTO, E., CANNAZZA, G. et al. (2018). "TDR-based measurements of water content in construction materials for in-the-field use and calibration", IEEE Transactions on Instrumentation and Measurement, 67(5): 1230-1237. https://doi. org/10.1109/TIM.2017.2770778
ČERNÝ, R. (2009). "Time-domain reflectometry method and its application for measuring moisture content in porous materials: A review", Measurement, 42(3): 329-336. https://doi.org/10.1016/j. measurement.2008.08.011

FORT, R., ÁLVAREZ DE BUERGO, M., VÁZQUEZ CALVO, M.C. et al. (2015). Caracterización de sales en el interior de la muralla de Ávila para su monitorización (Informe inédito). Instituto de Geociencias (CSIC-UCM). Grupo de Petrología Aplicada a la Conservación del Patrimonio. Laboratorio de Petrofísica.

FREITAS, T.S., GUIMARAES, A.S., ROELS, S. et al. (2020). Time Domain Reflectometry (TDR) technique - A solution to monitor moisture content in construction materials. E3S Web of Conferences, 172. https://doi.org/10.1051/e3sconf/202017217001

GARCÍA MORALES, S., LÓPEZ GONZÁLEZ, L. y COLLADO GÓMEZ, A. (2012). "Metodología de inspección higrotérmica para la determinación de un factor de intensidad de evaporación en edificios históricos", Informes de la Construcción, 64: 69-78. https:// doi.org/10.3989/ic.11.073

GIL-MUÑOZ, M.T. (2017). Análisis e interpretación de los datos de monitorización del lienzo 44-45 de la muralla de Ávila, tras su intervención para prevenir las humedades. Primer semestre de mediciones (Informe inédito). Ayuntamiento de Ávila.

HAUSCHILD, T. \& MENKE, F. (1998). "Moisture measurement in masonry walls using a non-invasive reflectometer", Electronics Letters, 34(25): 2413-2414. https://doi.org/10.1049/el:19981694

HEALY, W.M. (2003). "Moisture sensor technology - A summary of techniques for measuring moisture levels in building envelopes", ASHRAE Transactions, 109(1): 232-242.

HOŁA, A., MATKOWSKI, Z. \& HOŁA, J. (2017). "Analysis of the moisture content of masonry walls in historical buildings using the basement of a medieval town hall as an example", Procedia Engineering, 172: 363-368. https://doi.org/10.1016/j. proeng.2017.02.041

KAATZE, U. \& HUBNER, C. (2010). “Electromagnetic techniques for moisture content determination of materials", Measurement Science and Technology, 21. https://doi.org/10.1088/0957$\underline{0233 / 21 / 8 / 082001}$

KRUS, M. (1996). Moisture transport and storage coefficients of porous mineral building materials. Theoretical principles and new test methods. Ph.D.Thesis, Fraunhofer Institute of Building Physics.

LA VANGUARDIA. (1/3/2020). "Las visitas a la muralla de Ávila aumentaron casi el $50 \%$ en febrero". La Vanguardia. https://www. lavanguardia.com. [consulta: 26/4/2021].

MARTÍNEZ GARRIDO, M.I., FORT GONZÁLEZ, R. y RUIZ GONZÁLEZ, M. (2015). Aportación de la monitorización mediante redes de sensores y técnicas no invasivas para la conservación preventiva del Patrimonio. Tesis doctoral, Universidad Politécnica de Madrid. 
MCCANN, D.M. \& FORDE, M.C. (2001). "Review of NDT methods in the assessment of concrete and masonry structures", NDT\&E International, 34(2): 71-84. https://doi.org/10.1016/S0963$\underline{8695(00) 00032-3}$

MOLLO, L. y GRECO, G. (2011). “Moisture measurements in masonry materials by time domain reflectometry", Journal of Materials in Civil Engineering, 23(4): 441-444. https://doi.org/10.1061/(ASCE) MT.1943-5533.0000188

PAVLÍK, Z. \& ČERNÝ, R. (2004). "Application of TDR measurement technology for construction materials in semi-scale experiments", International Agrophysics, 18(3): 249-252.

PHILLIPSON, M.C., BAKER, P.H., DAVIES, M. et al. (2008). “Suitability of time domain reflectometry for monitoring moisture in building materials", Building Services Engineering Research and Technology, 29(3): 261-272. https://doi.org/10.1177/0143624408092423

PHILLIPSON, M., BAKER, P., MCNAUGHTAN, A. et al. (2007). "Moisture measurement in building materials: An overview of current methods and new approaches", Building Services Engineering Research and Technology, 28 (4): 303-316. https://doi. org/10.1177/0143624407084184

PINCHIN, S.E. (2008). "Techniques for monitoring moisture in walls", Reviews in Conservation, 9: 33-45. https://doi.org/10.1179/ sic.2008.53.Supplement-2.33

REGALADO, C.M., RITTER, A. y GARCÍA, O. (2009). "Caracterización de sensores electromagnéticos de humedad de suelo con fluidos estándar de permitividad conocida". En Estudios de la Zona no Saturada del Suelo, Silva Rojas, O. y Carrera Ramírez, J. (eds.). Barcelona: CIMNE, 192-199.

RUIZ, R. y CABRERA, B. (2017). "Arqueología en la Muralla de Ávila: últimas aportaciones", Cuadernos de arquitectura y fortificación, 4: 9-46.

SAÏD, A.N.M. (2007).“Measurement methods of moisture in building envelopes-A literature review", International Journal of Architectural Heritage, 1:293-310. https://doi.org/10.1080/15583050701476754

SANDROLINI, F. \& Franzoni, E. (2006). "An operative protocol for reliable measurements of moisture in porous materials of ancient buildings", Building and Environment, 41(10): 1372-1380. https:// doi.org/10.1016/j.buildenv.2005.05.023

SUCHORAB, Z., SOBCZUK, H. \& ŁAGÓD, G. (2016). "Estimation of building material moisture using non-invasive TDR sensors". In AIP Conference Proceedings, 1752. https://doi.org/10.1063/1.4955231

TADA, S. \& WATANABE, K. (1998). "An overview of principles and techniques of moisture properties measurement for building materials and components", Workshop on mass-energy transfer and deterioration of building materials and components, Tsukuba, Japan.

TIANO, P. \& RIMINESI, C. (2017). "State of arts of monumental stones diagnosis and monitoring", International Archives of the Photogrammetry, Remote Sensing and Spatial Information Sciences, 42: 665-671. https://doi.org/10.5194/isprs-archives-XLII2-W5-665-2017

TORRES, M.I.M. \& FREITAS, V.P. (2010). "The influence of the thickness of the walls and their properties on the treatment of rising damp in historic buildings", Construction and Building Materials, 24: 13311339. https://doi.org/10.1016/j.conbuildmat.2010.01.004

VECCHIATTINI, R. (2018). "Moisture monitoring experience in the old town of Genoa (Italy)", Journal of Cultural Heritage, 31S: 71-81. https://doi.org/10.1016/j.culher.2018.04.007

VOUTILAINEN, J. (2005). Methods and instrumentation for measuring moisture in building structures. Doctoral Thesis, Helsinki University of Technology, Applied Electronics Laboratory.

ZHAO, J.H., RIVERA, E., MUFTI, A. et al. (2012).“Evaluation of dielectric based and other methods for moisture content measurement in building stones", Journal of Civil Structural Health Monitoring, 2: 137 148. https://doi.org/10.1007/s13349-012-0024-1

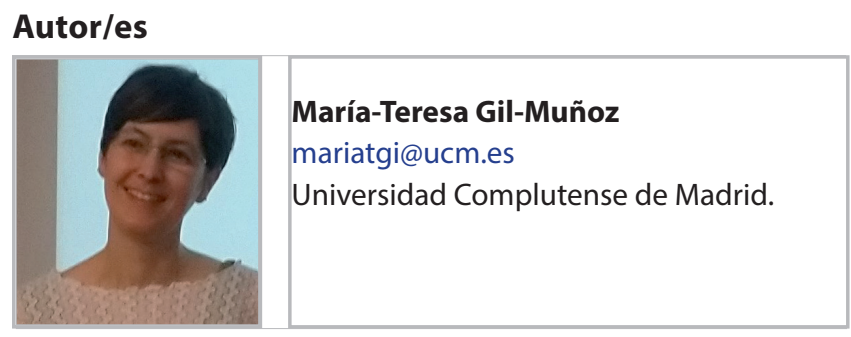

Diplomada en restauración y licenciada en arquitectura, es doctora por la Universidad Politécnica de Madrid, donde es invitada del grupo de investigación "Análisis e Intervención en el Patrimonio Arquitectónico". Es especialista en el ámbito de la conservación preventiva del patrimonio cultural y en el diagnóstico de humedades en edificios históricos. Compagina su trabajo profesional con la docencia en la Universidad Complutense de Madrid. Ha trabajado para instituciones como el Instituto del Patrimonio Cultural de España y ha realizado distintos estudios y propuestas de conservación en colecciones y monumentos relevantes de la Península Ibérica, elaborando planes de conservación preventiva, estudios de condiciones ambientales y diagnosis de humedades. También participa como docente en fundaciones, colegios profesionales y otras universidades. https://orcid.org/0000-0003-3674-2711

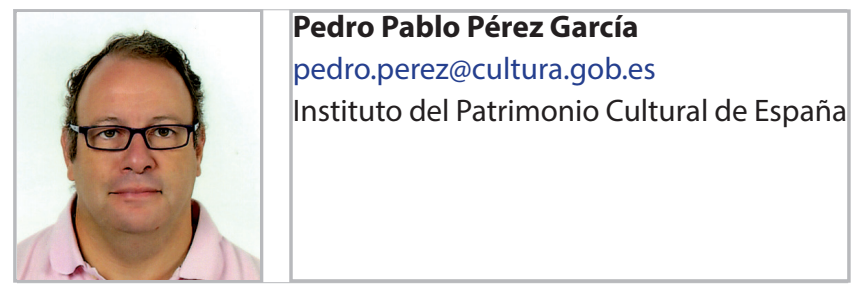

Geólogo del Instituto de Patrimonio Cultural de España, es especialista en petrología aplicada en la restauración de monumentos, habiendo participado en estudios analíticos de más de 400 monumentos. Estos estudios han supuesto 
la caracterización de materiales pétreos, morteros, cerámica, vidrio, depósitos salinos, recubrimientos y materiales pictóricos. $\mathrm{Ha}$ colaborado en numerosos proyectos de investigación con el Instituto de Patrimonio Cultural de España así como con otras instituciones públicas, fundaciones y empresas privadas. También ha participado en numerosos planes directores. Ha sido profesor asociado de la Universidad de Salamanca. Ha publicado de manera individual o con otros autores más de 30 publicaciones relacionadas con estudios analíticos en patrimonio y ha dado más de 50 conferencias y ponencias en distintos cursos, máster y congresos. Entre las técnicas analíticas que maneja habitualmente, cabe destacar la microscopía electrónica, la microscopía óptica, la difracción de Rayos-X y la termografía de infrarrojos.

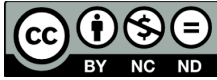

https://doi.org/10.37558/gec.v19i.997 